\title{
POSIBLES MECANISMOS DE ACCIÓN BIOLÓGICA DE LA ACTIVIDAD FÍSICA EN EL CÁNCER DE MAMA
}

\author{
POSSIBLE BIOLOGICAL MECHANISMS OF ACTION OF PHYSICAL ACTIVITY ON BREAST \\ CANCER
}

Jairo Alejandro Fernández Ortega* José Antonio de Paz Fernández**

\section{Resumen}

La evidencia científica ha demostrado la importancia de la actividad física como una estrategia efectiva para disminuir el riesgo y la recidiva del cáncer de mama. Sin embargo, hasta el momento no se conocen con precisión los mecanismos de acción biológicos del ejercicio sobre el cáncer de mama. El propósito de este artículo es realizar una revisión sistemática exhaustiva de la literatura e identificar las posibles hipótesis que se plantean de los mecanismos de acción biológicos del ejercicio sobre el cáncer de mama. Se realizó una revisión sistemática de la literatura publicada desde 1990 hasta 2011 sobre los efectos del ejercicio en el cáncer de mama.

Palabras clave: cáncer de mama, mecanismos de acción biológicos, actividad física, factores de riesgo, ejercicio físico.

\section{Abstract}

Scientific evidence has demonstrated the importance of physical activity as an effective strategy to reduce risk and relapse of breast cancer. However, the exact biological mechanisms of action of physical exercise on breast cancer are not known yet. The purpose of this paper is to carry out an exhaustive systematic review of the literature associated to this topic, and identify the possible hypothesis proposed to the mechanisms of biological action of physical exercise on breast cancer. Method: Direct a systematic literature review published from 1990 to 2011 about the effects of physical exercise on woman with breast cancer.

Keywords: breast cancer, mechanisms of biological action, physical activity, risk factors, physical exercise.

Fecha de recepción: 12 de diciembre de 2013

Fecha de aprobación: 28 de marzo de 2014

Docente de planta, Universidad Pedagógica Nacional de Colombia. Laboratorio de Fisiología del Ejercicio, Bogotá Colombia. Correo electrónico: jairofdz@pedagogica.edu.co

${ }^{* *}$ Doctor en Medicina y Cirugía. Especialista en Medicina Deportiva. Profesor titular, Departamento de Fisiología. Universidad de León (España). Correo electrónico: japazf@unileon.es 


\section{INTRODUCCIÓN}

El cáncer de mama es una de las neoplasias malignas más frecuentes en la mujer. Es el segundo cáncer más habitual y el más común: 4,7 millones de diagnosis y 502.000 muertes anuales en el mundo (Marxfeld, Staedtlerb y Harleman, 2006) de las cuales más de la mitad de los casos ocurren en países industrializados. Los datos más recientes de la Agencia Internacional de Investigaciones sobre el Cáncer (IARC, por su sigla en inglés) con relación a la morbilidad mundial del cáncer de mama corresponden a 2002; en ese año, el IARC calcula que se diagnosticaron aproximadamente 1.150.000 nuevos casos y hubo aproximadamente 411.000 defunciones por esta causa (Parkin, 2011).

Este incremento en la morbimortalidad en las mujeres, condujo a la búsqueda no solo de las posibles causas sino de mecanismos de prevención. De esta manera surge la actividad física como un factor asociado a la disminución del riesgo de cáncer de mama.

La actividad física es un factor con gran potencial para modificar el riesgo del cáncer de mama y se ha asociado a un riesgo disminuido entre mujeres premenopáusicas y posmenopáusicas (McTiernan, 2003). La idea de la relación entre actividad física y cáncer no es nueva; Rammazzini hace 300 años sugirió que la actividad física cumplía un papel en la etiología del cáncer humano (Thune y Furberg, 2001), y en 1922 aparecen los primeros estudios epidemiológicos que indican el rol de la actividad física en la prevención del cáncer (Lee, 2003).

A partir de la última década, los investigadores alentados por los resultados con estudios con modelos animales consolidaron la relación actividad física y riesgo de cáncer. En la actualidad existe suficiente evidencia epidemiológica y experimental del rol que cumple la actividad física como mecanismo de prevención primario en la reducción del riesgo de cáncer de mama (HoffmanGoetz, Apter, Demark-Wahnefried, Goran, McTiernan y Reichman, 1998; Lahmann, Friedenreich, Schuit, Salvini, Allen, Key y Riboli, 2007; McNeely, Campbell, Rowe, Klassen, Mackey y Courneya, 2006). La Fundación Mundial de Investigación en Cáncer (WCRF, por su sigla en inglés) y el Instituto Americano de Investigaciones en Cáncer (AICR, por su sigla en inglés), desarrollaron un estudio observacional sobre la evidencia epidemiológica de la asociación entre ejercicio físico y cáncer de mama. En el proyecto se revisaron 44 estudios de los cuales
32 presentan evidencia de disminución del riesgo, con un rango de 0,3-1,6, y un porcentaje entre el 30 y 40\% (World Cancer Research Fund y American Institute for Cancer Research, 2007). Loprinzi, Cardinal, Smit y Winters-Stone (2012) realizaron una revisión de la literatura y sobre un total de 76 estudios, identificaron que 40 de ellos (53\%) reportaron una relación estadísticamente significativa del efecto positivo que tiene el ejercicio sobre el riesgo de cáncer de mama; 28 (37\%) no reportaron disminuciones significativas del riesgo, y 8 (10\%) no registran evidencia de asociación.

Diversos estudios de cohorte, de caso control, revisiones sistemáticas y metaanálisis que se han realizado en los últimos veinte años ratifican esta relación estableciendo un rango de riesgo estimado de 0,3-1,6 y un porcentaje de reducción de riesgo entre el 30 y $40 \%$ (Bardia et al., 2006; Bernstein, Henderson, Hanisch, Sullivan-Halley y Ross, 1994; Coogan, Newcomb, Clapp, Trentham-Dietz, Baron y Longnecker, 1997; Dorgan, Brown, Barrett, Splansky, Kreger, D’Agostino y Schatzkin, 1994; Friedenreich y Cust, 2006; Friedenreich y Orenstein, 2002; Friedenreich y Rohan, 1995; Gammon,,Schoenberg, Britton, Kelsey, Coates, Brogan, Potischman, Swanson, Daling, Stanford, Brinton, 1998; Lee., 2003; Margolis,Mucci, Braaten, Kumle, Lagerros, Adami, Lund, Weiderpass, 2005; McTiernan, 2003; Rockhill, Willett,, Hunter 1999; Schwartz, 2008; Sesso, Paffenbarger y Lee, 1998; Shoff Newcomb, TrenthamDietz, Remington, Mittendorf, Greenberg, Willett, 2000; Thune, Brenn, Lund y Gaard, 1997; Thune, 2001) y del 40-80\% (Monninkhof, 2007; Adams, 2006; Bernstein, Henderson, Hanisch, Sullivan-Halley, y Ross, 1994; Carpenter, Ross,Paganini-Hill y Bernstein, 1999; D’Avanzo, Nanni, La Vecchia, Franceschi, Negri, y Giacosa, 1996; Fraser y Shavlik, 1997; Friedenreich, 2008; Friedenreich y Rohan, 1995; Frisch, Wyshak, Albright, Albright, Schiff, y Witschi, 1985; Hirose,Tajima, Hamajima, Inoue, Takezaki, Kuroishi y Tokudome1995.; Levi, Pasche, Lucchini y La Vecchia, 1999; Marcus, P. M.; Newman, B.; Moorman, P. G.; Millikan, R. C.; Baird, D. D.; Qaqish, B. y Sternfeld, B.1999; A McTiernan, Stanford, Weiss, Daling, \& Voigt, 1996; Mezzetti, La Vecchia, Decarli, Boyle, Talamini, y Franceschi. 1998; Mittendorf, Longnecker, Newcomb, Dietz, Bogdan, y Willett,1995.; Moradi, NyreÂn, Zack, Magnusson, Persson y Adami, 2000;Mutrie, Campbell, Whyte, McConnachie, Emslie, Lee, y Ritchie, 2007; Rockhill, 1999; 
Ueji, Ueno, Osei-Hyiaman, Takahashi y Kano, 1998; Verloop, Rookus, Van der Kooy y Leeuwen, 2000; Wyshak y Frisch, 2000; Zheng, Shu, McLaughlin, J. K.; Chow, W.-H.; Gao, Y. T. y Blot, W. J. et al., 1993). Sin embargo, algunos estudios no han divulgado ningún beneficio (Moore, Falson ,Mink, Hong, Anderson. y Kushi 2000) o incremento en el riesgo (Bardia, 2006).

Sin embargo, a pesar, de la evidencia científica, la relación entre actividad física y cáncer de mama es compleja, debido a que no se ha podido establecer claramente los mecanismos de acción biológicos del ejercicio físico sobre el cáncer de mama y la relación dosis/ respuesta. Por otra parte, se encuentran inconsistencias en los resultados de dicha asociación que pueden ser debido en muchos casos a limitaciones metodológicas, forma de evaluar los niveles de actividad física de los sujetos, control de los efectos de la modificación, complejidad y multiplicidad de los mecanismos biológicos y la dificultad metodológica para su captura y seguimiento (Friedenreich, 2001).

\section{METODOLOGÍA}

Para lograr comprender los mecanismos de acción biológicos del ejercicio sobre el cáncer de mama, se desarrolló una revisión de las diferentes publicaciones que sobre el tema se han publicado en los últimos quince años, a partir de una búsqueda sistemática. Se incluyeron ensayos controlados aleatorios y no aleatorios de ejercicio físico, revisiones y metaanálisis. Se incluyeron ensayos con diagnóstico de cáncer de mama de cualquier tipo de estadio, al igual que todas las formas de ejercicio, tipos de duración, intensidad y frecuencia. Se utilizaron varias estrategias de indagación; se realizaron búsquedas manuales en las revistas especializadas y búsquedas en el registro especializado de ensayos controlados del grupo Cochrane de Cáncer de Mama (Cochrane Breast Cancer Specialised Register, 16 de julio de 2004) y en las bases de datos electrónicas de Medline, PubMed, Proquest, Hinari, SportDiscus, EMBASE, Ebsco Host CancerLit, CINAHL, PsychINFO, PEDro y Ovid, y las páginas de la OPS, OMS, CDC de Atlanta y CELAFISCS de Brasil. Igualmente, se revisaron tesis doctorales o disertaciones de ensayos clínicos. Además, se buscaron las listas de referencia de todos los estudios potencialmente relevantes y se entró en contacto con expertos y autores de estudios anteriores, para identificar los artículos relevantes (Fernández y De Paz, 2012).

\section{ESTUDIOS EXPERIMENTALES DE LOS MECANISMOS BIOLÓGICOS DEL CÁNCER DE MAMA A PARTIR DE MODELOS ANIMALES}

Los estudios epidemiológicos humanos no aportan información detallada acerca de la iniciación y progresión del cáncer, en relación con el ejercicio físico. Por esta razón, los modelos con animales son herramientas importantes para investigar la patogénesis y para desarrollar las estrategias del tratamiento para la metástasis del cáncer en seres humanos. Los modelos animales de cáncer de mama en ratas han sido utilizados ampliamente en los últimos cuarenta años para estudiar la susceptibilidad al cáncer, ayudar a identificar nuevos genes mamarios, descifrar los mecanismos de la resistencia al cáncer mamario; como modelo para intentar comprender el proceso (etiología, evolución y metástasis) las características biológicas morfológicas, bioquímicas y la lesión molecular del cáncer; el impacto de fármacos en el tratamiento, y recientemente como modelo para comprender el tipo de actividad física y el impacto que ella produce en los cancerígenos (Hoffman-Goetz., 2003 ; Thompson, 2006).

El tumor de mama que se forma en las ratas es similar al de los humanos (Marxfelda, 2006; Ravoori, 2007; Szpirer, 2007) Las ratas, ratones, perros y gatos desarrollan a menudo carcinoma mamario espontáneo. La evidencia científica indica que el desarrollo del carcinoma mamario en la rata tiene una evolución similar al cáncer de mama humano, así como también una similitud en la morfología de la glándula (Thompson, 2000), y en los biomarcadores tumorales.

Estos estudios, junto con los de carácter experimental realizados en seres humanos, han permitido establecer una serie de hipótesis que permiten identificar los posibles mecanismos de acción biológicos del ejercicio sobre el cáncer de mama e intentan igualmente responder a una serie de preguntas cruciales en la comprensión de esta relación como: ¿Cuál es el biomarcador ideal, o la dosis de actividad física biológicamente eficaz? ¿Cuál es el tipo de ejercicio que se debe realizar para obtener un efecto protector? ¿Existen periodos críticos para obtener un efecto protector? ¿Cuál es el tiempo de exposición adecuado? (Hoffman-Goetz et al., 1998; Rundle, 2005). 


\section{HIPÓTESIS SOBRE LOS POSIBLES MECANISMOS DE ACCIÓN BIOLÓGICOS DE LA ACTIVIDAD FÍSICA SOBRE EL CÁNCER DE MAMA}

Se presume que la actividad física tiene influencia sobre los posibles mecanismos biológicos que intervienen en el desarrollo del cáncer de mama como el balance energético, entorno hormonal, sistema inmunitario y reparación del ADN. A su vez, el impacto de este efecto está determinado por la intensidad, duración, frecuencia y tipo de ejercicio (Thune y Furberg, 2001). En la figura 1 se presentan varios de los posibles factores de riesgo primario y secundario de cáncer de mama (Irwin, 2006), y en la tabla 1, se presenta la forma como la actividad física ejerce su acción sobre esos posibles mecanismos biológicos.
Figura 1. Hipótesis de la relación entre actividad física y factores primarios y secundarios de riesgo de cáncer de mama.

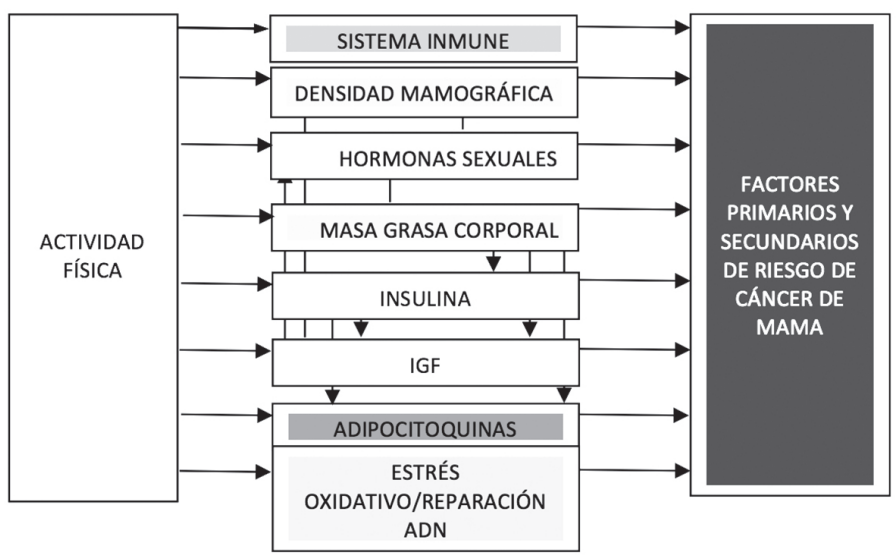

Tomado de: Irwin (2006).

Tabla 1.

\begin{tabular}{|c|c|c|}
\hline \multicolumn{3}{|c|}{ Posibles mecanismos de acción biológicos de la actividad física sobre el cáncer de mama } \\
\hline Posibles mecanismos involucrados & $\begin{array}{l}\text { Factor de riesgo para el cáncer de } \\
\text { mama, asociado al mecanismo }\end{array}$ & $\begin{array}{l}\text { Efectos de la actividad física } \\
\text { sobre el mecanismo }\end{array}$ \\
\hline \multirow{5}{*}{ Disminución de las hormonas sexuales } & \multirow[b]{2}{*}{ Incremento en la proliferación celular } & Retardo en la menarquia \\
\hline & & $\begin{array}{l}\text { Reducción del número de } \\
\text { ciclos ovulatorios }\end{array}$ \\
\hline & \multirow{3}{*}{ Disminución de la apoptosis } & $\begin{array}{c}\text { Disminución en la producción } \\
\text { de estrógenoovárico }\end{array}$ \\
\hline & & $\begin{array}{l}\text { Reducción de los lipidosproductores } \\
\text { de estrógenos }\end{array}$ \\
\hline & & $\begin{array}{c}\text { Incremento en la SHGB lo que conduce a } \\
\text { una disminución en la biodisponibilidad } \\
\text { de los estrógenos y la testosterona }\end{array}$ \\
\hline \multirow{3}{*}{ Disminución de la masa grasa corporal } & Grasas almacenamiento de carcinógenos & $\begin{array}{c}\text { Disminución de los depósitos viscerales } \\
\text { y subcutáneos de grasa corporal }\end{array}$ \\
\hline & Incremento hormonas sexuales & \\
\hline & Aumento en los niveles de insulina & \\
\hline \multirow{4}{*}{ Reducción de insulina y IGFs } & \multirow{3}{*}{ Incremento hormonas sexuales } & $\begin{array}{l}\text { Incremento posreceptor } \\
\text { señalizador de la insulina }\end{array}$ \\
\hline & & $\begin{array}{l}\text { Aumento en trasportador } \\
\text { de glucosa y ARNm }\end{array}$ \\
\hline & & $\begin{array}{l}\text { Incremento en el aclaramiento } \\
\text { de ácidos grasos libres }\end{array}$ \\
\hline & Incremento en la proliferación celular & $\begin{array}{l}\text { Aumento en la liberación de } \\
\text { glucosa al musculo }\end{array}$ \\
\hline
\end{tabular}




\begin{tabular}{|c|c|c|}
\hline \multirow{4}{*}{ Incremento de la función inmune } & \multirow{4}{*}{ Reconoce y elimina células anormales } & $\begin{array}{c}\text { Cambios en la composición muscular para } \\
\text { favorecer la utilización de la glucosa }\end{array}$ \\
\hline & & $\begin{array}{l}\text { Incrementa el número y la } \\
\text { actividad de los macrófagos }\end{array}$ \\
\hline & & $\begin{array}{l}\text { Aumento las linfoquinas que } \\
\text { activa la muerte celular }\end{array}$ \\
\hline & & $\begin{array}{l}\text { Incremento en la proliferación } \\
\text { de linfocitos }\end{array}$ \\
\hline \multirow{2}{*}{ Disminución de las adipocitoquinas } & Promueve angiogénesis & \multirow{2}{*}{$\begin{array}{l}\text { Disminuye TNF } \alpha \text {, leptina, CRP, y IIL-6 y } \\
\text { aumento de adiponectinavia disminución } \\
\text { de la grasa corporal y la insulina }\end{array}$} \\
\hline & Estimula la biosíntesis de estrógeno & \\
\hline $\begin{array}{l}\text { Disminución de la densidad } \\
\text { mamográfica }\end{array}$ & Incremento en la proliferación celular & $\begin{array}{l}\text { Disminuye las hormonas sexuales, } \\
\text { insulina e IGF, que a su vez puede } \\
\text { disminuir la densidad mamográfica. }\end{array}$ \\
\hline $\begin{array}{l}\text { Mejora de los sistemas de } \\
\text { defensa antioxidante }\end{array}$ & $\begin{array}{l}\text { Radicales libres producen } \\
\text { daños en el ADN }\end{array}$ & $\begin{array}{l}\text { Mejora las defensas contra radicales libres } \\
\text { y la reparación del ADN, por el incremento } \\
\text { en los niveles de enzimas antioxidantes. }\end{array}$ \\
\hline $\begin{array}{l}\text { SHBG: glob } \\
\text { TNF } \alpha \text { : fact }\end{array}$ & $\begin{array}{l}\text { adora de hormonas sexuales. IGF: insulina } \\
\text { ecrosis tumoral. CRP: proteína C reactiva. }\end{array}$ & $\begin{array}{l}\text { tor de crecimiento. } \\
\text { : interleuquina } 6 .\end{array}$ \\
\hline
\end{tabular}

Tomado de: Irwin (2006).

\section{IMPACTO EN EL MANTENIMIENTO DEL BALANCE ENERGÉTICO}

Uno de los posibles mecanismos biológicos que tiene el ejercicio físico es el control de la composición corporal a través del balance energético. El balance energético es un concepto importante en la exploración de la etiología de numerosos enfermedades crónicas, incluido el cáncer de mama, por la estrecha relación entre la ganancia de peso, (sobrepeso) y el incremento en el riesgo de cáncer de mama (Christine M Friedenreich \& Orenstein, 2002).

Un balance energético positivo conduce al incremento del tejido adiposo que se traduce en una ganancia de peso, cuya distribución no es homogénea y, por ejemplo, la grasa visceral que se encuentra alrededor de los órganos puede generar un mayor riesgo de cáncer de mama. En el tejido adiposo se almacenan toxinas, medicamentos y ciertas vitaminas, y su almacenamiento se constituye en una continua fuente de cancerígenos (McTiernan, Ulrich, Slate y Potter, 1998).
El balance energético positivo puede generar varios efectos que pueden ser de forma directa o a través del incremento del tejido graso como: insulinorresistencia y sus consecuencias como la hiperinsulinemia y la hipertrigliceridemia (Ballard-Barbash, Birt, Kestin y King, 1997; Bruning et al., 1992); incremento en los niveles de IGF-I y otros factores de crecimiento; aumento en el número total de ciclos ovulatorios. El aumento del peso corporal se asocia con una edad más temprana de la menarquia y edad más tardía de la menopausia.

Por otra parte, el exceso de masa grasa puede producir efectos sobre las concentraciones plasmáticas de las hormonas esteroides, como aumento en los niveles de estrógenos activos, debido al efecto que tiene el tejido adiposo sobre la circulación hormonal, puesto que sirve como fuente extragonadal para la conformación de estrógenos desde el precursor adrenal de andrógenos -conversión de androstenediona a estradiol, por acción de las aromatasas-. 
Este balance energético positivo también puede conducir a Bajos niveles de SHBG lo cual conlleva a un incremento en los niveles de estradiol libre y activo (Hoffman-Goetz et al., 1998).

El ejercicio físico tiene un efecto positivo en el balance energético, que se manifiesta en la disminución del tejido adiposo, lo cual conduce a modificar los efectos nocivos que fueron descritos anteriormente (McTiernan, Ulrich, Slate, Potter, 1998; Thune y Furberg, 2001). La actividad física tiene un efecto similar a la restricción de la dieta, sobre la glándula adrenal incrementando la excreción urinaria de esteroides corticales inmunorreactivos y los niveles de corticosterona en orina, lo cual tiene una asociación inversa con la multiplicación del carcinoma mamario (Thompson, Zhu y Jiang, 2004).

\section{INSULINA: SU INFLUENCIA Y SU PAPEL COMO FACTOR DE CRECIMIENTO.}

Existe suficiente evidencia experimental y epidemiologia del rol que tiene la IGF-I en diversos tipos de cáncer (Ryan y Goss, 2008). Están claramente establecidas varias de las acciones que el IGF-I y IGF-II estimulan a nivel celular y que favorecen el desarrollo del tumor por su carácter mitogénetico, como la proliferación celular y la inhibición de la apoptosis en muchos tipos de tejido, incluyendo células de cáncer de mama (Canzian et al., 2006; Espelund, Cold, Frystyk, Rskov y Flyvbjerg, 2008).

También se puede afirmar que la IGF-I e IGF-II actúan indirectamente como indicadores de la actividad de la hormona sexual en las mujeres jóvenes, o también puede ser que interactúan directamente con los estrógenos para aumentar el riesgo de padecer cáncer de mama. De hecho, se ha demostrado que el estradiol acentúa la expresión de la IGF-I y los receptores de ambas hormonas, dando como resultado una interacción sinérgica que estimula la proliferación de células de cáncer de mama (Allen et al., 2005).

Altos niveles circulatorios de IGF-I y bajos niveles de (IGFBP-III) están asociados a un incremento en el riesgo de cáncer de mama (Friedenreich, 2001; McTiernan, Kooperberg, White y Coll, 2003; McTiernan, Ulrich, Slate y Potter, 1998).

Elevadas concentraciones sanguíneas de insulina estimulan la producción de IGF-I, y reducen las concentraciones plasmáticas de SHBG, eventos que están relacionados con un incremento en el riesgo de cáncer de mama.
En su forma más simple, la hipótesis insulina/cáncer postula que un estado de hiperinsulinemia prolongado reduce las proteínas transportadoras de IGF-I, las IGFBP, que tienen como función inhibir su biodisponibilidad. Este evento conduce a incrementos en los niveles del IGF-I bioactivo libre, y cambios concomitantes en el ambiente celular que favorece el desarrollo del tumor (Renehan, Harvie y Howell, 2006).

Altos niveles de actividad física pueden reducir el riesgo del cáncer de mama a través de la vía de señalización de la insulina. El ejercicio reduce la circulación de insulina y glucosa, lo cual puede afectar los niveles de IGF-I e IGFBP (McTiernan, Ulrich, Slate y Potter, 1998) lo que conduce a una disminución de la hiperinsulemia e insulinorresistencia, que podría dar lugar a una disminución en la biodisponibilidad de IGF-I y, por tanto, reduce el riesgo de cáncer de mama (Adams et al., 2006). Sin embargo, el efecto del ejercicio es más específico sobre IGF y sus proteínas de unión (IGFBP-III).

Adicionalmente, al reducir los niveles de insulina se incrementa la circulación de la globulina transportadora de hormonas sexuales (SHBG) de tal forma que se reduce la biodisponibilidad del estradiol y la testosterona (Neilson, Friedenreich, Brockton, \& Millikan, 2009). En la figura 2.se describen estos mecanismos (Renehan et al., 2006).

\section{EFECTO SOBRE LAS HORMONAS SEXUALES}

Está claramente establecido que las hormonas endógenas sexuales (estradiol, progesterona) están implicadas fuertemente en la etiología del cáncer de mama, por su participación en el desarrollo y crecimiento del tumor, estimulando la mitosis de las células epiteliales y regulando la progresión del ciclo celular (Emaus et al., 2008). En las mujeres, elevados niveles de estrógenos y andrógenos incrementan el riesgo de cáncer de mama. Los andrógenos están implicados en el desarrollo del tumor, debido a que se convierten en estrógenos en el tejido adiposo por acción de las aromatasas (Atkinson et al., 2004; Friedenreich y Cust, 2008).

Varios estudios de cohorte (Rundle, 2005) han divulgado asociaciones estadísticamente significativas entre los niveles de estrógenos y andrógenos endógenos, y el riesgo de cáncer de mama en mujeres posmenopáusicas. Aunque no se conoce con precisión los 
Figura 2. Eje insulina- IGF

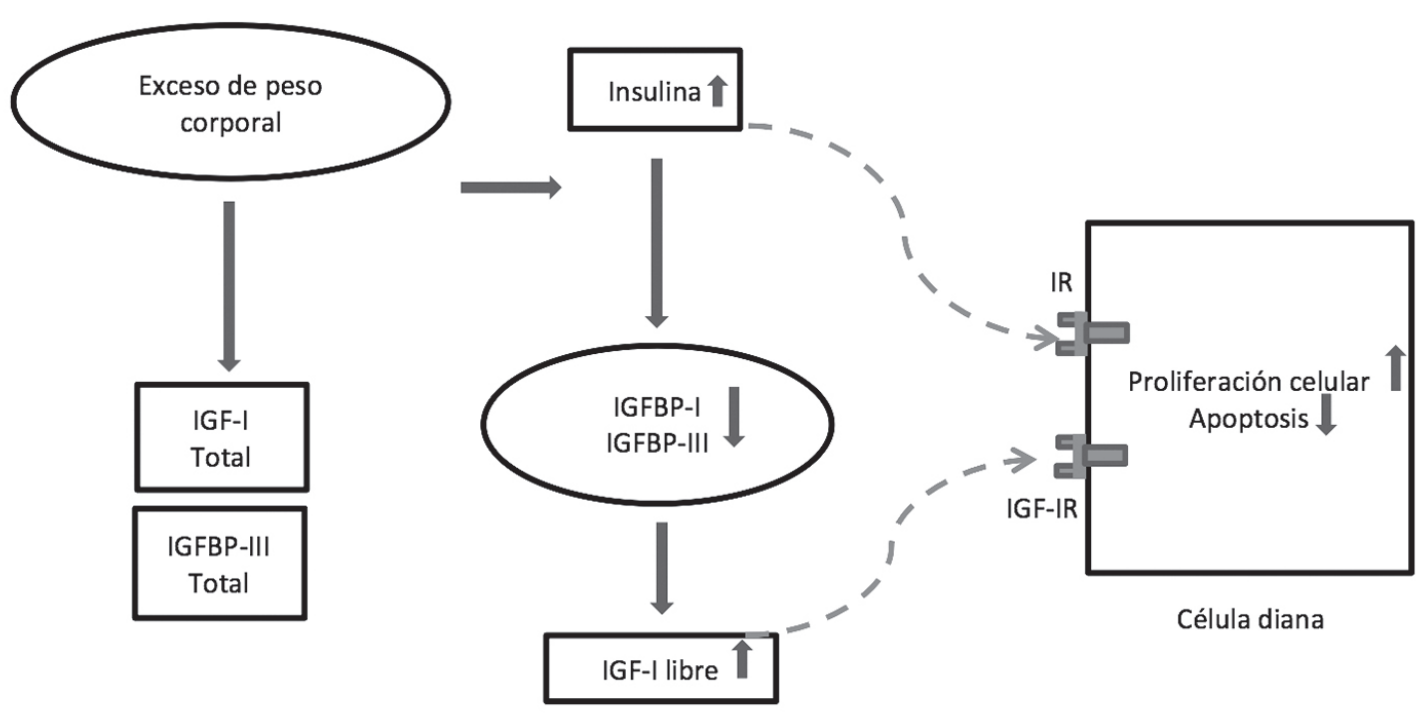

Tomado de: Renehan, A. Roberts, D. Dive, C. Obesity and cancer: Pathophysiological and biological mechanisms

mecanismos, estos pueden variar según la fase de la vida reproductiva y dependen de interacciones con numerosos factores etiológicos.

La evidencia científica sobre la exposición hormonal indica una relación entre el número total de ciclos menstruales y el riesgo de cáncer de mama, razón por la cual cuando la menarquia se presenta a muy temprana edad se incrementa el riesgo. Por el contrario, las mujeres que a lo largo de su vida hayan tenido ciclos menstruales irregulares (por tanto, reducción en las concentraciones de estradiol y progesterona) parecen tener una reducción del 50\% de riesgo de cáncer mama en comparación con las que tuvieron ciclos regulares (Hoffman-Goetz et al., 1998).

El metabolismo de los estrógenos en los seres humanos está mediado principalmente por las enzimas del citocromo P450, y dos de los principales metabolitos hidroxilados de la estrona: 2-hidroxiestrona (2-OH E1) y 16a-hydroxyestrona (16a-OH E1) que presentan propiedades diferentes. La 2-OH E1 es débilmente estrogénica y se ha sugerido que es anticancerígena. Por el contrario, 16a-OH E1 es más estrogénica, pues forma enlaces covalentes con los receptores de estrógenos y puede ser genotóxica. Se ha reportado riesgo en el incremento de cáncer de mama asociado con una baja la excreción urinaria de 2-OH E1 en relación con 16-OH E1 (Atkinson et al., 2004).
En el estudio "La actividad física para la salud total", McTierman et.al. (1999) compararon el efecto en el perfil de las hormonas sexuales en mujeres sedentarias de un entrenamiento aeróbico de moderada intensidad, contra uno de fuerza. Se identificó un efecto positivo en el ejercicio aeróbico en la relación de la excreción urinaria de 2-OH E1 y 16a-OH E1. Estos resultados también se presentaron en el estudio de Atkinson et al. (2004), quienes sometieron a entrenamiento a un grupo de mujeres, durante doce semanas y encontraron diferencias estadísticamente significativas para los cambios en la relación de excreción urinaria de 2-OH E1 y 16a-OH E1, cuando se relacionó con la disminución de la grasa intraabdominal, en las mujeres que perdieron desde 15 centímetros en adelante en el perímetro abdominal.

El ejercicio intenso y la pérdida del peso conducen a un cambio en el metabolismo del estrógeno que desencadena una hidroxilación del C2- y C4. Los metabolitos que resultan de este proceso 2- y 4 hidroxiestrogenos, que debido a su estructura química híbrida (en parte catecolamina, en parte esteroide) se agrupan generalmente como catecolestrógenos, ejercen funciones mediadoras cruciales en el hipotálamo y la pituitaria (Timmer y De Cre'e, 2008). Por otra parte, la actividad física, junto con el balance energético son moduladores del eje hipotálamo/hipófisis/ovario, lo cual genera una serie de eventos de cascada metabólica que pue- 
den regular la producción endógena, metabolismo, excreción, capacidad de enlace de estas hormonas y conversión periférica, y de esta forma reducir los niveles biológicamente disponibles (Hoffman-Goetz, 1998; Thune, 2001).

Se plantea que hay dos caminos principales a partir de los cuales la actividad física podría reducir la exposición a los estrógenos y otras hormonas endógenas. Primero, la actividad física ayuda a mantener el balance energético y a reducir la grasa corporal y, por tanto, la reducción de los estrógenos (Rockhill et al., 1999). Los estudios observacionales y de intervención en mujeres posmenopáusicas indican que la asociación inversa entre los niveles de estrógeno y los de actividad física son atribuibles en parte al decrecimiento de IMC.

El segundo camino indica que el ejercicio especialmente de alta intensidad reduce los niveles hormonales circulatorios endógenos, al igual que la exposición acumulativa a la hormona por alteraciones en la función menstrual como: retraso en el inicio de la menarquia; irregularidad o ausencia en los periodos menstruales; función anormal luteinizante por pérdida de movilidad de la hormona; un ciclo menstrual prolongado y bajos niveles de progesterona y estradiol (Friedenreich y Cust, 2008; Friedenreich y Orenstein, 2002).

Se ha demostrado que la actividad física altera el entorno hormonal en mujeres premenopáusicas probablemente aumentando los niveles de catecolaminas y b-endorfinas, que inhiben la secreción hipotalámica de la hormona gonadotropina coriónica; disminuyendo la síntesis y secreción de las hormonas folículo estimulante y LH, lo cual conduce a reducir la producción y secreción de estrógenos y de progesterona. También la actividad física puede aumentar los niveles de SHBG (Friedenreich, 2001; McTiernan et al., 2003; McTiernan, Ulrich, Slate y Potter, 1998).

\section{ALTERANDO LA RESPUESTA INFLAMATORIA}

Los indicadores de inflamación están constituidos por un grupo de compuestos formados por las adipoquinasleptina, adiponectina, TNF- $\alpha$ y IL-6 que son un grupo de polipéptidos biológicamente activos producidos por lo adipocitos en el tejido adiposo, y la proteína $C$ reactiva que no es una adipoquina pero se produce en el hígado como respuesta a los niveles de TNF- $\alpha$ y IL-6.

La inflamación crónica está considerada como un factor de riesgo para varios tipos de cáncer, entre ellos el de mama. Diversos mecanismos biológicos implican a las adipoquinas en la etiología del cáncer de mama, pero la medida en que estas influyen de forma directa en el riesgo de cáncer o en los biomarcadores está sin resolver (Neilson et al., 2009).

Las adipocitoquinas presentan fuertes asociaciones con el índice de masa corporal, masa grasa abdominal, e hiperinsulinemia. Además, varios adipocitoquinas, incluyendo la interleuquina 6 (IL-6), TNF- $\alpha$, y la leptina, promueven la angiogénesis, que es esencial para el desarrollo y progresión del cáncer de mama y puede estimular la biosíntesis de estrógenos por la inducción de la actividad de la aromatasa (Irwin, 2006).

Se ha demostrado que elevados niveles de actividad física están asociados a niveles inferiores de marcadores de inflamación, el estrógeno ha mostrado ser proinflamatorio, y otros estrógenos como el 17 betaestradiol poseen una función inhibidora de la expresión del gen inflamatorio, que es regulado por el factor nuclear $\mathrm{kB}$ (McTiernan et al., 2003; McTiernan, Ulrich, Slate y Potter, 1998; Slattery et al., 2007).

En la revisión de literatura realizada por Neilson et al. (2009), y que se resume en la tabla 2, se presenta el posible rol que tiene cada uno de estos componentes en el desarrollo del cáncer de mama en mujeres posmenopáusicas y el impacto que podría tener la actividad física. 
Tabla 2.

\begin{tabular}{|c|c|c|}
\hline $\begin{array}{l}\text { Biomarcadores } \\
\text { propuestos }\end{array}$ & $\begin{array}{l}\text { Posible rol en el cáncer de mama en } \\
\text { mujeres posmenopáusicas }\end{array}$ & $\begin{array}{l}\text { Posible impacto de la actividad física } \\
\text { en las mujeres posmenopáusicas }\end{array}$ \\
\hline Leptina & $\begin{array}{l}\text {-Induce las aromatasas y estabiliza los } \\
\text { receptores- } \alpha \text { de estrógeno. } \\
\text {-Aunque la leptina puede mejorar la sensibilidad a } \\
\text { la insulina, niveles elevados de leptina se asocian } \\
\text { con resistencia a la insulina. Las acciones de la } \\
\text { leptina a nivel hipotalámico podrían disminuir } \\
\text { teóricamente la sensibilidad sistémica a la } \\
\text { insulina y la producción de adiponectina. } \\
\text { - La expresión es inducida por altos } \\
\text { niveles de estrógenos e insulina. } \\
\text { - Mitógeno en las células del cáncer de mama; } \\
\text { inhibe la apoptosis; proangiogénico. }\end{array}$ & $\begin{array}{l}\text { La pérdida de peso reduce la grasa } \\
\text { corporal, que es la fuente prin- } \\
\text { cipal de leptina circulante }\end{array}$ \\
\hline Adiponectina & $\begin{array}{l}\text {-La expresión génica y la secreción de los adipocitos } \\
\text { se reducen por el TNF-a y la IL-6, la producción tam- } \\
\text { bién puede ser reducida parcialmente por la leptina. } \\
\text {-Promueve y mejora la sensibilidad a la insulina; la adi- } \\
\text { ponectina conduce a la reducion de la resistencia a } \\
\text { la insulina y la hiperinsulinemia compensatoria. } \\
\text {-Antiangiogénica; antimitogenético y antiinflamato- } \\
\text { rio. En una línea de las células del cáncer de mama } \\
\text { la adiponectina no tuvo ningún efecto sobre la apop- } \\
\text { tosis, pero sí inhibió la proliferación celular. }\end{array}$ & $\begin{array}{l}\text { La pérdida de grasa disminuye la IL-6 } \\
\text { y TNF-a, que son potentes inhibidores } \\
\text { de la expresión y la secreción de adipo- } \\
\text { nectina. Por tanto, la pérdida de peso } \\
\text { puede aumentar los niveles circulan- } \\
\text { tes de adiponectina. La actividad física } \\
\text { crónica puede reducir la inflamación, } \\
\text { por ejemplo, la circulación de IL-6, } \\
\text { TNF- } \alpha \text { independientemente de la pér- } \\
\text { dida de grasa, sin embargo, los mecanis- } \\
\text { mos de este efecto son desconocidos. }\end{array}$ \\
\hline TNF- $\alpha$ & $\begin{array}{l}\text { - Regulador clave de la IL-6 síntesis. } \\
\text { - Estimula la biosíntesis de estrógenos a través } \\
\text { de la inducción de las aromatasas. } \\
\text {-Induce la insulinorresistencia. } \\
\text { - Acción paradójica: inhibe la proliferación de células } \\
\text { tumorales y actúa también como promotor del tumor. } \\
\text { Pude causar daños directos en el ADN; antiapoptótica } \\
\text { y mitogénica, promueve la invasión, la angiogénesis } \\
\text { y la metástasis de las celular tumoral. }\end{array}$ & $\begin{array}{l}\text {-La pérdida de grasa puede disminuir } \\
\text { los niveles de TNF- } \alpha \text { dado que el ARNm } \\
\text { de TNF- } \alpha \text { y TNF- } \alpha \text { proteina, son libera- } \\
\text { das del tejido adiposo en la obesidad. } \\
\text { - La actividad física crónica puede } \\
\text { reducir el número de células mono- } \\
\text { nuclares en la sangre con lo que } \\
\text { se agota una fuente de TNF- } \alpha \text {. }\end{array}$ \\
\hline
\end{tabular}




\begin{tabular}{|c|c|c|}
\hline IL-6 & $\begin{array}{l}\text { - Liberación es estimulada por TNF- } \alpha \text {;se ha especulado que } \\
\text { la IL-6 producción se refleja durante la producción de TNF- } \\
\alpha \text {; a su vez, la IL-6 ejerce efectos inhibitorios sobre el TNF- } \alpha \text {. } \\
\text { - Cumple un papel primordial en la estimula- } \\
\text { ción hepática para la producción de CRP. } \\
\text {-Produce insulinorresistencia en los adipósitos: otra } \\
\text { posible fuente del su rol en la diabetes tipo II. } \\
\text { - Estimula la biosíntesis de estrógeno por la induc- } \\
\text { ción en la actividad de las aromatasas. } \\
\text { - Promueve la motilidad de células del cáncer de } \\
\text { mama que sugiere un papel en la metástasis. } \\
\text { - Papel complejo de IL-6 en las células de cán- } \\
\text { cer de mama in vitro. Regula las proteínas antia- } \\
\text { poptóticas y angiogénicos de las células tumora- } \\
\text { les, y también induce la apoptosis en el carcinoma } \\
\text { de mama positivo para receptores de estrógenos }\end{array}$ & $\begin{array}{l}\text { - A pesar que han estudiado amplia- } \\
\text { mente los efectos agudos del ejer- } \\
\text { cicio sobre los niveles de IL-6, los } \\
\text { mecanismos mediante el cual la } \\
\text { actividad física crónica altera los } \\
\text { niveles de IL-6 no son claros. } \\
\text { - La adiposidad reducida puede dismi- } \\
\text { nuir los niveles de IL-6, dado que es una } \\
\text { de las fuentes donde se origina las IL-6. } \\
\text { - La actividad física crónica puede } \\
\text { reducir el número de células mono- } \\
\text { nucleares en la sangre con lo que } \\
\text { se agota una fuente de IL-6. }\end{array}$ \\
\hline CRP & $\begin{array}{l}\text { - Es un marcador típico de inflamación. } \\
\text { - Su producción es estimulada por las TNF- } \alpha \text { e IL-6. } \\
\text { - Está independientemente asociado con la lep- } \\
\text { tina en sujetos sanos, posiblemente por la } \\
\text { inducción de la IL-6 por la leptina. } \\
\text { - La producción de CRP está fuertemente ligada con la resis- } \\
\text { tencia a la insulina y puede cambiar con los niveles de insu- } \\
\text { lina independientemente de los cambios en la obesidad. }\end{array}$ & $\begin{array}{l}\text { A largo plazo la actividad física reduce la } \\
\text { CRP por reducción de los adipositos, por } \\
\text { reducción en la producción de citoqui- } \\
\text { nas (IL-6 y TNF- } \alpha \text { ) en las células mono- } \\
\text { nucleares celulares y por otros medios. }\end{array}$ \\
\hline
\end{tabular}

Tomado de: Neilson et al., (2009).

\section{SISTEMA INMUNE}

La evidencia científica sugiere que la mayoría de cánceres humanos no son de orden inmunológico, sin embargo, estos son susceptibles en cierto grado al control de varios mecanismos del sistema inmune innato, como por ejemplo las linfoquinas activador de las células asesinas NK, macrófagos de tumores infiltrantes, y por los productos secretados de estas células como las citosinas y eicosanoides. Las reacciones inmunitarias innatas a las células cancerosas son un aspecto importante de la defensa del huésped, por ello se cree que el sistema inmunológico desempeña un papel importante en la protección contra el cáncer de mama, mediante el reconocimiento y la eliminación de células anormales (Irwin, 2006; Hoffman-Goetz et al., 1998).

El sistema inmune innato está constituido por componentes celulares, factores solubles, barreras físicas y sistema reticuloendotelial. Es la primera línea de defensa contra agentes patógenos mientras se activa la respuesta inmune. El sistema inmune reconoce los antígenos tumorales con el fin de generar una respuesta de las células T. La detección y destrucción de células tumorales para la defensa contra el cáncer están mediadas por los mecanismos de cada uno de los 
componentes del sistema inmune innato. En cuanto al componente celular, los mecanismos implicados comprenden: las células (NK) activadas por linfoquinas (LAK); los macrófagos que constituyen una defensa de primera línea contra el CA y son capaces de disminuir la incidencia, progresión y metástasis de algunos tumores; los neutrófilos y células asesinas naturales, y nivel de los factores solubles la proteína C-reactiva, la interleucina 1 (IL-1), el factor de necrosis tumoral (TNF- $\alpha$ ) y el interferón (IFN) (Fairey, Courneya, Field y Mackey, 2002b).

El ejercicio representa una forma de estrés para la homeostasis del organismo lo que puede inducir respuestas neurohormonales, metabólicas y cardiorrespiratorias, íntimamente ligadas a la respuesta inmune. La evidencia científica indica que el ejercicio físico afecta a una variedad de parámetros inmunes naturales, tanto numérica como funcionalmente. Muchos de estos efectos son transitorios y reflejan cambios hemodinámicos con el ejercicio agudo tales como: el reclutamiento de poblaciones de linfoides del tejido de reserva, un efecto endocrino inmediato que incluye la liberación de catecolaminas, glucocorticoides y hormonas opiáceas (Hoffman-Goetz et al., 1998).

En los últimos veinte años, los efectos del ejercicio sobre la cantidad y función de varios de los componentes del sistema inmune han sido bien descritos y revisados con regularidad (Mackinnon, 1997; Rowbotton y Green, 2000; Nieman, 1997; Shephard y Shek, 1999). Sin embargo, a pesar de la evidencia, todavía quedan muchas preguntas por resolver sobre el efecto de la actividad física en el sistema inmune, y particularmente frente a la intensidad, duración y frecuencia del ejercicio. Una de las hipótesis sugiere que el ejercicio moderado mejora la función del sistema inmune y reduce la susceptibilidad al cáncer, por el contrario ejercicios de alta intensidad generan una supresión del sistema inmune y elevan la susceptibilidad al cáncer, aunque los estudios no han demostrado el rol del sistema inmune en la carcinogénesis (McTiernan et al., 2003; McTiernan, Ulrich, Slate y Potter, 1998; Tiernan et al., 2003.; Westerlind, 2003).

Por el contrario, estudios realizados en animales con ejercicio de alta intensidad demuestran un mejor efecto de este tipo de ejercicio que el de baja y moderada intensidad sobre el sistema inmune innato (Fairey et al., 2002b).

Lúdica pedaqóqica. No. 19 (2014 - I)

pp.57 - 72
En general, durante e inmediatamente después del ejercicio físico se presenta un incremento superior al 140\% en el número de leucocitos en el plasma en proporción a la intensidad y duración del ejercicio. De la misma manera, el ejercicio genera efectos agudos y crónicos sobre la expresión génica de neutrófilos y linfocitos, por lo tanto es un potente estímulo para aumentar el número de linfocitos y la expresión génica (Rowbotton y Green, 2000). En el periodo posterior al ejercicio se presenta una disminución en el número de linfocitos y monocitos a niveles inferiores a los del reposo, (Gabriel, Schwarz y Kindermann, 1992; Nieman y Donohue, 1991) pero el número de neutrofilos circulantes continúa aumentando horas después del ejercicio (Fry, Morton, Crawford y Keast, 1992; McCarthy y Dale, 1988). Se cree que esta linfocitopenia durante el periodo posterior se presenta en ejercicios prolongados o de alta intensidad (Rowbotton y Green, 2000).

En varios estudios realizados con pacientes con cáncer de mama se ha observado un efecto positivo del ejercicio físico en el sistema inmune de estas pacientes, tales como disminución en el tiempo de la neutropenia y la trombopenia (Dimeo, Fetscher, Lange, Mertelsmann y Keul, 1997), en la actividad de las células naturales asesinas (Peters, Lötzerich, Niemeier, Schüle y Uhlenbruck, 1994; Nieman et al., 1995; Na, Kim, Kim, Ha y Yoon, 2000), aumento en la cantidad de granulocitos, y disminución de linfocitos y monocitos, pero con aumento en la capacidad fagocítica de los monocitos (Peters, Lötzerich, Niemeir, Schüle y Uhlenbruck, 1995).

Los ejercicio de corta duración y moderada intensidad causan perturbaciones en el número de leucocitos circulantes hasta casi 60 minutos después de finalizado el ejercicio y el estado de homeostasis retorna varias horas después dependiendo de la duración y la intensidad del ejercicio (Gabriel, Schwarz, Steffens y Kindermann, 1992; Gabriel y Kindrman 1997; Hansen, Wilsgard y Osterud, 1991; McCarthy, Grant y Marbut, 1991) Los mecanismos de estas leucocitosis todavía no están bien dilucidados. Sin embargo, existen algunas evidencias que indican a las catecolaminas y al cortisol como mediadores de ese proceso.

Otra hipótesis plantea que la actividad física incrementa el metabolismo de la detoxificación, generada por las especies reactivas y la química de la carcinogénesis, disminuyendo así la exposición al daño del 
ADN. La evidencia científica apunta a que la actividad física incrementa la reparación del ADN, lo cual a su vez reduce su daño y por tanto la iniciación del tumor. Se sugiere que la actividad física reduce los niveles de factor de crecimiento, lo cual conduciría a reducir la proliferación (la cual genera errores en la replicación) y por consiguiente dismminuir la iniciación. Adicionalmente bajos niveles de factor de crecimiento están asociados con una reducción en la iniciación, promoción y progresión del tumor. Por esta razón el incremento en los niveles de ciertas hormonas aumenta el riesgo (Rundle, 2005).

Por otra parte, conforme con la hipótesis que los factores hormonales concomitantes al ejercicio modulan la actividad de la muerte celular natural, las conclusiones son que los receptores b-adrenérgicos implicados en la muerte celular natural pueden ser regulados por el ejercicio ya que la liberación de adrenalina durante el ejercicio conduce al incremento en el número o en la actividad de la muerte celular natural, por acción de las linfoquinas, citoquinas, neutrofilos, y la circulación aumentada de linfocitos, granulositos, monocitos y macrófagos (Hoffman-Goetz et al., 1998). Estos eventos potencializan la capacidad del sistema inmune para retardar la tasa de crecimiento y de lisis de las células tumorales. Igualmente, el ejercicio tiene un efecto potencial sobre la función inmune a través de la mediación de las hormonas sexuales, debido al efecto inmunorregulador que ellas ejercen sobre: el subconjunto de células $\mathrm{T}$, los macrófagos, la síntesis de inmunoglobulinas, y diversas citoquinas que son el blanco de las hormonas sexuales (McTiernan, Ulrich, Slate y Potter, 1998). La progesterona y los andrógenos parecen suprimir el sistema inmune, mientras que los estrógenos pueden ser estimulantes o supresores, dependiendo de la situación.

La relación entre la actividad física, el sistema inmune natural y el riesgo de cáncer de mama puede estar asociada a la exposición a estrógenos. Varias investigaciones en estudios in vitro han demostrado que altas concentraciones y exposición a estrógenos conducen a una reducción en la actividad de las NK (Hanna y Schneider, 1983; Seaman y Gindhart, 1979). Sin embargo esta relación sigue siendo una hipótesis especulativa.

\section{CONCLUSIONES}

De las evidencias observadas en los diferentes estudios, se puede plantear que existe una serie de posibles caminos biológicos a través de los cuales la actividad física podría ejercer un impacto positivo en la disminución del riesgo de cáncer de mama. Sin embargo, las posibles rutas son complicadas, numerosas y pueden estar afectadas por otra serie de factores como los hábitos de vida, lo que no ha permitido determinar realmente su impacto.

Dentro de la evidencia del efecto de la actividad física en el cáncer de mama hay algunos aspectos en que concuerdan la mayoría de los estudios. El primero es el efecto que tiene la actividad física en la disminución del tejido adiposo, lo cual disminuye el riesgo de cáncer de mama posiblemente a través de un mecanismo de cascada, por el efecto que este tiene a nivel de la insulinorresistencia y en las hormonas sexuales. En segunda instancia, también está claramente demostrado cómo la práctica de actividad física regular y de alta intensidad desempeña un papel crucial en la disminución de exposición a los estrógenos, lo cuales han sido indicados como factores de riesgo de cáncer de mama. Frente a los efectos del ejercicio físico sobre los factores de inflamación e inmunes hay todavía bastante controversia.

El otro tema central de la discusión es la dosis respuesta. En la actualidad se sabe que la magnitud de las respuestas hormonales, biológicas y adaptativas al ejercicio físico están determinadas por la intensidad, duración, frecuencia y tipo de ejercicio.

La diversidad metodológica utilizada en cada uno de los estudios frente a estos parámetros, no permite establecer con claridad, cual es la intensidad, duración y tipo de ejercicio más apropiado para lograr un efecto positivo sobre cada uno de posibles mecanismos.

Por estas razones, se requiere un mayor número de estudios experimentales que permitan comprender con mayor precisión cómo operan los mecanismos biológicos de la actividad física en la disminución del riesgo del cáncer de mama, y que determinen de forma precisa la dosis respuesta, para de esta forma lograr realizar recomendaciones de actividad física que permitan la reducción del riesgo, la recurrencia y mejoren la calidad y esperanza de vida de estas mujeres. 


\section{REFERENCIAS BIBLIOGRÁFICAS}

Adams, S.A. et al. (2006). Association of physical activity with hormone receptor status: the Shanghai Breast Cancer Study. Cancer Epidemiol Biomarkers Prev., 15, 1170-1178.

Allen, N.: Allen, D.; Fentiman, I.; Dos Santos Silva, I.; Peto, J.; Holly, J. y Key, T. (2005). A prospective study of serum insulin-like growth factor-I (IGF-I),IGF-II, IGFbinding protein-3 and breast cancer risk. Br J Cancer, 92, 1283-1287.

Atkinson, C.; Lampe, J. W.; Tworoger, S. S.; Ulrich, C. M.; Bowen, D.; Irwin, M. L. y McTiernan, A. (2004). Effects of a moderate intensity exercise intervention on estrogen metabolism in postmenopausal women. Cancer Epidemiol Biomarkers Prev 13(5), 868-874.

Ballard-Barbash, R.; Birt, D. F.; Kestin, M. y King, I. B. (1997). Perspectives on Integrating Experimental and Epidemiologic Research on Diet, Anthropometry and Breast Cancer. The Journal of Nutrition 127(5), 936S-939S.

Bardia A., H. L., Hartmann, L, Vachon, C, Vierkant, R. A.,Wang, A.H.,Olson, J. E. Sellers, T.A.Cerhan, J. R. (2006). Recreational Physical Activity and Risk of Postmenopausal Breast Cancer Based on Hormone Receptor Status. Arch Intern Med., 166, 2478-2483.

Bernstein, L.; Henderson, B.; Hanisch, R.; Sullivan-Halley, J. y Ross, R. (1994). Physical exercise and reduced risk of breast cancer in young women. J Natl Cancer Inst, 86, 1403-1408.

Bruning, P. F.; Bonfrèr, J. M. G.; Van Noord, P. A. H.; Hart, A. A. M.; De Jong-Bakker, M. y Nooijen, W. J. (1992). Insulin resistance and breast-cancer risk. International Journal of Cancer 52(4), 511-516. DOI: 10.1002/ijc.2910520402

Canzian, F.; McKay, J. D.; Cleveland, R. J. Biessy, C; Rinaldi, S; Landi, S; Boillot, C; Monnier, S;Chaje`s, V; Clavel-Chapelon, F; Te'hard, B y Chang-Claude, T. (2006). Polymorphisms of genes coding for insulinlike growth factor 1 and its major binding proteins, circulating levels of IGF-I and IGFBP-3 nd breast cancer risk: results from the EPIC study Br J Cancer (94), 299 - 307.

Carpenter, C.; Ross, R.; Paganini-Hill, A. y Bernstein, L. (1999). Lifetime exercise activity and breast cancer risk among post-menopausal women. Br J Cancer, 80, (11), 1852-1858.

Coogan, P. F.; Newcomb, P. A.; Clapp, R. W.; Trentham-Dietz, A.; Baron, J. A. y Longnecker, M. P. (1997.). Physical activity in usual occupation and risk of breast cancer (United States). Cancer Causes \& Control, 8, 626-631.
D’Avanzo, B.; Nanni, O.; La Vecchia, C.; Franceschi, S.; Negri, E. y Giacosa, A. (1996). Physical activity and breast cancer risk. Cancer. Epidemiol Biomarkers Prev., $5,155-160$.

Dimeo, F.; Fetscher, S.; Lange, W.; Mertelsmann, R. y Keul, J. (1997). Effects of aerobic exercise on the physical performance and incidence of treatment-related complications after high-dose chemotherapy. Blood, 90(1), 3390-3394.

Dorgan, J. F.; Brown, C.; Barrett, M.; Splansky, G. L.; Kreger, B. E.; D’Agostino, R. B. y Schatzkin, A. (1994). Physical activity and risk of breast cancer in the Framingham heart study. Am. J. Epidemiol., 139, 662-669.

Emaus, A.; Veierkd, M. B.; Furberg, A.-S.; Espetvedt, S.; Friedenreich, C. M.; Ellison, P. T. y Thune, I. (2008). Physical activity, heart rate, metabolic profile, and estradiol in premenopausal wome. Med Sci Sports Exerc 08, 1022-1030.

Espelund, U.; Cold, S.; Frystyk, J.; Rskov, H. y Flyvbjerg, A. (2008). Elevated free IGF2 levels in localized, earlystage breast cancer in women. Eur J Endocrinol, 159, 595-601.

Fairey, A. S.; Courneya, K. S.; Field, C. J. y Mackey, J. R. (2002a). Physical exercise and immune system function in cancer survivors. A comprehensive review and future directions. Cancer 94(2), 539-551.

Fairey, A. S.; Courneya, K. S.; Field, C. J. y Mackey, J. R. (2002b). Physical exercise and immune system function in cancer survivors. A comprehensive review and future directions. Cancer 94,(2), 539-551.

Fernández, J. A. y De Paz, J. A. (2012). Cáncer de mama y ejercicio físico: Revisión. Hacia la Promoción de la Salud, 17,(1), 135-153.

Fraser, G. E. y Shavlik, D. (1997). Risk factors, lifetime risk, and age at onset of breast cancer. Ann. Epidemiol, 7, 375-382.

Friedenreich, C. (2001). Physical Activity and Cancer Prevention: From Observational to Intervention Research1. Cancer Epidemiology, Biomarkers \& Prevention 10, 287-301.

Friedenreich C M, C. A. E. (2008). Physical activity and breast cancer risk: impact of timing, type and dose of activity and population subgroup effects. $\mathrm{Br}$. J. Sports Med., 42, 636-647.

Friedenreich, C. M. y Cust, A. E. (2008). Physical activity and breast cancer risk: impact of timing, type and dose of activity and population subgroup effects. Br. J. Sports Med., 42, 636-647. 
Friedenreich, C. M. y Orenstein, M. R. (2002). Physical Activity and Cancer Prevention Etiologic Evidence and Biological Mechanisms. J Nutr., 132, 3456-3464.

Friedenreich, C. M. y Rohan, T. E. (1995). Physical activity and risk of breast cancer. Eur.J. Cancer Prev, 4, 145-151.

Frisch, R. E.; Wyshak, G.; Albright, N. L.; Albright, T. E.; Schiff, I. y Witschi, J. (1985.). Lower prevalence of breast cancer and cancers of the reproductive system among former college athletes compared to nonathletes. Br. J. Cancer, 52, 885-891.

Fry, R.; Morton, A.; Crawford, G. y Keast, D. (1992). Cell numbers and in vitroresponses of leukocytes and lymphocyte subpopulations following maximal exercise and interval training sessions of different intensities. Eur. J. Appl. Physiol. Occup. Physiol, 64, 218-227.

Gabriel, H.; Schwarz, L.; Steffens, G. y Kindermann, W. (1992). Immunoregulatory hormones, circulating leukocyte and lymphocyte subpopulations before and after endurance exercise of different intensities. Int. J. Sports Med, 13, 359-366.

Gabriel, H. y Kinderman W. (1997). The acute immune response to exercise: what does it mean? Int. J. Sports Med., 18, S28-S45.

Gabriel, H. L.; Schwarz, P. B. y Kindermann, W. (1992). Differential mobilisation of leukocyte and lymphocyte subpopulations into the circulation during endurance exercise. Eur. J. Appl. Physiol. Occup. Physiol, 65, 529-534.

Gammon, M.; John, E., Britton,J. (1998). Recreational and Occupational Physical Activities and Risk of Breast Cancer J Natl Cancer Inst 90(2), 100-117.

Hanna, N. y Schneider, M. (1983). Enhancement of tumor metastasis and suppression of natural killer cell activity by b-estradiol treatment. J Immunol, 130, 974-980.

Hansen, J.; Wilsgard, L. y Osterud, B. (1991). Biphasic changes in leukocytes induced by strenuous exercise. Eur. J. Appl. Physiol. Occup. Physiol., 62, 157-161.

Hirose, K.; Tajima, K.; Hamajima, N.; Inoue, M.; Takezaki, T.; Kuroishi, T. y Tokudome, S. (1995.). A large-scale, hospital-based case-control study of risk factors of breast cancer according to menopausal status. Res. Jpn. J. Cancer, 86, 146-154.

Hoffman-Goetz, L.; Apter, D.; Demark-Wahnefried, W.; Goran, M. I.; McTiernan, A. y Reichman, M. E. (1998). Possible mechanisms mediating an association between physical activity and breast cancer. Cancer., 83(suppl), 621-628.

Hoffman-Goetz, L. (2003). Physical Activity and cancer prevention: Animal -tumor models. Med.Sci. sports Exerc, 35, 1828-1832.
Irwin, M. L. (2006). Randomized controlled trials of physical activity and breast cancer prevention. Exerc. Sport Sci. Rev 34(4), 182-193.

Lahmann, P. H.; Friedenreich, C.; Schuit, A. J.; Salvini, S.; Allen, N. E.; Key, T. J. y Riboli, E. (2007). Physical Activity and Breast Cancer Risk The European Prospective Investigation into Cancer and Nutrition. Cancer Epidemiol Biomarkers Prev 16(1), 36-42.

Lee., I. (2003). Physical activity and cancer prevention-data from epidemiologic studies. Med Sci Sports Exerc., 35(11), 1823-1827.

Levi, F.; Pasche, C.; Lucchini, F. y La Vecchia, C. (1999.). Occupational and leisure time physical activity and the risk of breast cancer. Eur. J. Cancer, 35, 775-778.

Loprinzi, P. D.; Cardinal, B. J.; Smit, E. y Winters-Stone, K. M. (2012). Physical activity and breast cancer risk. Journal of Exercise Science \& Fitness, 10, 1-7.

Mackinnon, L. T. (1997). Immunity in athletes. Int. J. Sports Med. 18, S62-68.

Marcus, P. M.; Newman, B.; Moorman, P. G.; Millikan, R. C.; Baird, D. D.; Qaqish, B. y Sternfeld, B. (1999). Physical activity at age 12 and adult breast cancer risk (United States). Cancer Causes \& Control, 10, 293-302.

Margolis, K.; Mucci L, Braaten T, Kumle, M; Adami, H.O; Lund, E y Weiderpass, E. (2005). Physical Activity in Different Periods of Life and the Risk of Breast Cancer: The Norwegian-Swedish Women's Lifestyle and Health Cohort Study. Cancer Epidemiol Biomarkers Prev, 14(1), 27-32.

Marxfeld, H.; Staedtlerb, F. y Harleman, J.H. (2006). Characterisation of two rat mammary tumour models for breast cancer research by gene expression profiling. Exp Toxicol Pathol, 58, 133-143.

Marxfeld, H.; Staedtlerb, F.; Harleman, J. (2006). Characterisation of two rat mammary tumour models for breast cancer research by gene expression profiling. Experimental and Toxicologic Pathology, 58, 133-143.

McCarthy, D. y Dale, M. (1988). The leucocytosis of exercise. Sports Med, 6, 333-363.

McCarthy, D. A.; Grant, M. y Marbut, M. (1991). Brief exercise induces an immediate and a delayed leukocytosis. Br. J. Sports Med, 25, 191-195.

McNeely, M. L.; Campbell, K. L.; Rowe, B. H.; Klassen, T. P.; Mackey, J. R. y Courneya, K. S. (2006). Effects of exercise on breast cancer patients and survivors: a systematic review and meta-analysis. CMAJ, 175(1), 34-41.

McTiernan, A. (2003). Behavioral risk factor en breast cancer:can risk be modifie. The oncologist, 8, 326-334. 
McTiernan, A.; Kooperberg, C.; White, E. Wilcox, S; Coates,R; Campbell, L; Woods,N y Ockene, J (2003). Recreational Physical Activity and the Risk of Breast Cancer in Postmenopausal Women: The Women's Health Initiative Cohort Study. JAMA 290(10), 1331-1336.

McTiernan, A.; Stanford, J.; Weiss, N.; Daling, J. y Voigt, L. (1996). Occurrence of breast cancer in relation to recreational exercise in women age 50-64 years. Epidemiology, 7, 598-604.

McTiernan, A.; Ulrich, C. M.; Yancey, D.; Slate, S.; Nakamura, H.; Oestreicher, N. y Schwartz, R. (1999). The Physical Activity for Total Health (PATH) Study: rationale and design. Medicine and science in sports and exercise, 31(9), 1307-1312.

McTiernan, A.; Ulrich, C.; Slate, S. y Potter J. (1998). Physical activity and cancer etiology: associations and mechanisms. Cancer Causes and Control, 9, 487-509.

Mezzetti, M.; La Vecchia, C.; Decarli, A.; Boyle, P.; Talamini, R. y Franceschi, S. (1998). Population attributable risk for breast cancer: diet, nutrition, and physical exercise. J. Natl. Cancer Inst., 90, 389394.

Mittendorf, R.; Longnecker, M. P.; Newcomb, P. A.; Dietz, A. T., Greenberg E. R, Bogdan, G. F. y Willett, W. C. (1995.). Strenuous physical activity in young adulthood and risk of breast cancer (United States). Cancer Causes \& Control, 6, 347-353.

Monninkhof, E.; Elias S.; Vlems F.; Schuit, A.; Voskuil, D.; y Van Leeuwen F. (2007). Physical activity and breast cancer: a systematic review. Epidemiology 18(1), 137-157.

Moore, D.B., Falson A.; Mink, P.J.; Hong, C.P.; Anderson, K.E. y Kushi LH. (2000). Physical activity and incidence of postmenopausal breast cancer. Epidemiology, 11, 292-296.

Moradi, T.; NyreÂn, O.; Zack, M.; Magnusson, C.; Persson, I. y Adami, H. (2000). Breast cancer risk and lifetime leisure-time and occupational physical activity (Sweden). Cancer Causes \& Control, 11, 523-531.

Mutrie, N.; Campbell, A. M.; Whyte, F.; McConnachie, A.; Emslie, C.; Lee, L. y Ritchie, D. (2007). Benefits of supervised group exercise programme for women being treated for early stage breast cancer:pragmatic randomised controlled trial. BMJ, 334, 517.

Na, Y.-M.; Kim, M.-Y.; Kim, Y.-K.; Ha, Y.-R. y Yoon, D. S. (2000). Exercise therapy effect on natural killer cell cytotoxic activity in stomach cancer patients after curative surgery. Archives of Physical Medicine and Rehabilitation 81(6), 777-779. DOI: http://dx.doi. org/10.1016/S0003-9993(00)90110-2
Neilson, H. K.; Friedenreich, C. M.; Brockton, N. T. y Millikan, R. C. (2009). Physical activity and postmenopausal breast cancer: Research proposed biologic mechanisms and areas for future. Cancer Epidemiol Biomarkers Prev 18(1), 11-27.

Nieman, D. (1997). Exercise immunology: practical applications. Int. J. Sports Med., 18, S91-S100.

Nieman, D. C.; Cook, V. D.; Henson, D. A.; Suttles, J.; Rejeski, W. J.; Ribisl, P. M. y Nehlsen-Cannarella, S. L. (1995). Moderate exercise training and natural killer cell cytotoxic activity in breast cancer patients. International journal of sports medicine 16(5), 334-337.

Nieman, D. C., L., N.-C. S. y Donohue, K. M. (1991). The effects of acute moderate exercise on leukocyte and lymphocyte subpopulations. Med. Sci. Sports Exerc, 23, 578-585.

Parkin, D. (2011). Cancers attributable to inadequate physical exercise in the UK in 2010. British Journal of Cancer, 105, S38-S41.

Peters, C.; Lötzerich, H.; Niemeier, B.; Schüle, K. y Uhlenbruck, G. (1994). Influence of a moderate exercise training on natural killer cytotoxicity and personality traits in cancer patients. Anticancer research 14(3A), 1033-1036.

Peters, C.; Lötzerich, H.; Niemeir, B.; Schüle, K. y Uhlenbruck, G. (1995). Exercise, cancer and the immune response of monocytes. Anticancer research, 15(1), 175-179.

Ravoori, S., V. M.; Sahoo, S.; Srinivasan, C. y Gupta, R.C. (2007). Mammary tumor induction in ACI rats exposed to low levels of 17 beta-estradiol. Int. J. Oncol 31(1), 113-120.

Renehan, A. G.; Harvie, M. y Howell, A. (2006). Insulinlike growth factor (IGF)-I, IGF binding protein-3, and breast cancer risk: eight years on. Endocrine-Related Cancer (13), 273-278.

Renehan, A G; Roberts y DL. Dive, C (2008). Obesity and cancer: Pathophysiological and biological mechanisms. Archives of Physiology and Biochemistry 114(1), 71-83.

Rockhill, B., Willett W, Hunter D, et al. (1999). Prospective Study of Recreational Physical Activityand Breast Cancer Risk. Arch Intern Med., 159, 2290-2296.

Rowbotton, D. G. y Green, K. J. (2000). Acute exercise effects on the immune system. Medicine \& Science in Sports \& Exercise 32(7), S396-S405.

Rundle, A. (2005). Molecular Epidemiology of Physical Activity and Cancer. Cancer Epidemiol Biomarkers Prev 14(1), 227-236. 
Ryan, P. D. y Goss, P. E. (2008). The Emerging Role of the Insulin-Like Growth Factor Pathway as a Therapeutic Target in Cancer. The Oncologist 13(1), 16-24. DOI: 10.1634/theoncologist.2007-0199

Schwartz, A. (2008). Physical activity. Semin Oncol Nurs 24(3), 164-170.

Seaman, W. E. y Gindhart, T. D. (1979). Effect of estrogen on natural killer cells. Arthritis \& Rheumatism 22(11), 1234-1240. DOI: 10.1002 /art.1780221110

Sesso, H. D., Paffenbarger, R. S. y Lee, I.-M. (1998). Physical activity and breast cancer risk in the College Alumni Health Study (United States). Cancer Causes \& Control, 9, 433-439.

Shephard, R. J. y Shek, P. N. (1999). Effects of exercise and training on natural killer cell counts and cytolytic activity: a meta-analysis. Sports Med., 28, 177-195.

Shoff, S. M.; Newcomb, P. A.; Trentham-Dietz, A.; Remington, P. L.; Mittendorf, R.; Greenberg, E. R. y Willett, W. C. (2000). Early-life physical activity and postmenopausal breast cancer: effect of body size and weight change. Cancer Epidemiol. Biomark. Prev., 9, 591-595, .

Slattery, M.L.; Edwards, S.; Murtaugh, M. A.; Sweeney, C.; Herrick, J.; Byers, T. y Baumgartner, K. B. (2007). Physical Activity and Breast Cancer Risk Among Women in the Southwestern United States. Annals of Epidemiology 17(5), 342-353.

Szpirer, C. y Szpirer,J. (2007). Mammary cancer susceptibility: human genes and rodent models. Mamm Genome, 18, 817-831.

Thompson, H. (2006). Pre-clinical investigations of physical activity and cancer: a brief review and analysis. Carcinogenesis 27(10), 1946-1949.

Thompson, H. J. (1994). Effect of exercise intensity and duration on the induction of mammary carcinogenesis. Cancer Research, 54, 1960-1963.

Thompson, H. J.; Zhu, Z. y Jiang, W. (2004.). Weight control and breast cancer prevention: Are the effects of reduced energy intake equivalent to those of increased energy expenditure? J. Nutr., 134, 3407S-3411S.

Thompson, H.S., M. (2000). Rat Models of Premalignant Breast Disease. Journal of Mammary Gland Biology and Neoplasia 5(4), 409-420.
Thune, I.; Brenn, T.; Lund, E. y Gaard, M. (1997). Physical activity and the risk of breast cancer. N. Engl. J. Med, $336,1269-1275$.

Thune I., F. A. (2001). Physical activity and cancer risk: dose-response and cancer, all sites and site-specific. Med Sci Sports Exerc. 33(6 suppl), S530-50, S609-610.

Thune, I. y Furberg, A.S. (2001). Physical activity and cancer risk: dose-response and cancer, all sites and site-specific. Med Sci Sports Exerc., 33((6 Suppl)), 530550.

Tiernan, A. et al. (2003.). Health Initiative Cohort Study Cancer in Postmenopausal Women. JAMA. 290(10), 1331-1336.

Timmer, E. C. y De Cre'e, C. (2008 ). Effect of energy deficiency on estrogen metabolist in female athletes. Med.Sci. Sports Exerc, 40, 397.

Ueji, M., Ueno, E., Osei-Hyiaman, D., Takahashi, H., \& Kano, K. (1998.). Physical activity and the risk of breast cancer: a case-control study of Japanese women. J.Epidemiol., 8, 116-122.

Verloop, J.; Rookus, M. A.; Van der Kooy, K. y Leeuwen, F. E. V. (2000). Physical Activity and Breast Cancer Risk in Women Aged 20-54 Years. J Natl Cancer Inst, 92(2), 128-135.

Westerlind, K. C. (2003). Physical activity and cancer prevention -mechanisms. Med Sci Sports Exerc. 35(11), 1834-1840. DOI: 10.1249/01. MSS.0000093619.37805.B7

World Cancer Research Fund y American Institute for Cancer Research (2007). Food, Nutrition, Physical Activity, and the Prevention of Cancer: a Global Perspective. Washington D.C.

Wyshak, G. y Frisch, R. E. (2000). Breast cancer among female college athletes compared to non-athletes: a 15-year follow-up. Br. J. Cancer, 82, 726-730.

Zheng, W.; Shu, X. O.; McLaughlin, J. K.; Chow, W.-H.; Gao, Y. T. y Blot, W. J. (1993). Occupational physical activity and the incidence of cancer of the breast, corpus uteri, and ovary in Shanghai. Cancer (Phila.), 71, 3620-3624,. 